\title{
ON A PROBLEM OF BRUCKNER AND CEDER CONCERNING THE SUM OF DARBOUX FUNCTIONS
}

\author{
J. S. LIPIŃSKI
}

\begin{abstract}
The main purpose of this paper is to show that for some continuous function $f$ and any preassigned, countable and dense set $D$ of real numbers there exists a measurable function $d$ which takes on every real value in every interval such that the range of $f+d$ is $D$.
\end{abstract}

A real valued function $f$ defined on an interval $I$ is said to have the intermediate value property if whenever $x_{1}$ and $x_{2}$ are in $I$, and $y$ is any number between $f\left(x_{1}\right)$ and $f\left(x_{2}\right)$, there is a number $x_{3}$ between $x_{1}$ and $x_{2}$ such that $f\left(x_{3}\right)=y$. Because of Darboux's work on this property, one now usually calls a function having the intermediate value property a Darboux function. A function $f$ is Darboux if and only if $f$ maps any connected subset of $I$ onto a connected set.

Since the sum of Darboux functions may fail to be a Darboux function, some mathematicians have examined how badly it can fail. Bruckner and Ceder [1] have recently shown that corresponding to each Darboux function $f$ which is not constant on any subinterval, there exists a function $d$ which takes on every real value in every subinterval of $I$ such that the range of $f+d$ is a preassigned countable and dense set. Certainly $d$ is a Darboux function and the range of $f+d$ is totally disconnected.

The technique used by Bruckner and Ceder in constructing the function $d$ does not imply the measurability of $d$. Indeed Bruckner and Ceder have shown that $d$ must be nonmeasurable whenever $f$ is absolutely continuous. They asked whether $d$ had to be nonmeasurable if we weaken absolute continuity of $f$ to continuity of $f$.

This paper answers this question affirmatively for certain continuous functions $f$. This is a consequence of the following.

THEOREM. Let $g$ be a Darboux function which is not constant on any subinterval of its domain I such that the set

$$
A=\left\{\alpha: \alpha \in R, g^{-1}(\alpha) \text { is perfect }\right\}
$$

Received by the editors March 2, 1976.

AMS (MOS) subject classifications (1970). Primary 26A15; Secondary 26A21, 28A20.

Key words and phrases. Darboux functions. 
is dense in $g(I)$. Then there exists a homeomorphism $h$ from I onto itself such that for every countable, dense set $D \subset R$ there exists a function $d$ which takes on every real value in every subinterval of $I$ such that $D$ is the range of $(g \circ h)+d$ and $(g \circ h)+d$ is constant almost everywhere.

It is known that there exist continuous functions $g$ which are not constant on any interval such that $A=g(I)$. See for example [4]. (The properties of the set $A$ for. a Darboux function $g$ are discussed in [2] and [3].) Let $g$ be such a function. Now define $f$ by $f=g \circ h$ where $h$ is the homeomorphism of the Theorem above. Then $f$ is continuous and from this Theorem the range of $f+d$ is $D$. By the Theorem $f+d$ is constant almost everywhere. Thus $d$ is measurable.

In order to prove the Theorem we shall use the type of construction suggested by Theorem 1 of [1]. But first we need the following.

LEMma. Suppose $F \subset I$, and for every interval $J \subset I$ there exists a perfect set $P \subset F \cap J$. Then there exists a homeomorphism $\chi$ from I onto itself such that $\chi(F)$ is measurable and $|\chi(F)|=|I|$.

Proof. The Lemma is trivial if $F=I$. Suppose that $I \backslash F \neq \varnothing$. Let $P^{0}$ be a perfect subset of $F$. Choose an increasing homeomorphism $h_{1}$ from $I$ onto itself so that $\left|h_{1}\left(P^{0}\right)\right|>\frac{1}{2}|I|$. Let $\left\{\left(a_{i}, b_{i}\right)\right\}$ be a sequence which may be finite, of disjoint open intervals, such that $I \backslash\left(\{a\} \cup\{b\} \cup h_{1}\left(P^{0}\right)\right)=\cup_{i}\left(a_{i}, b_{i}\right)$. Choose a sequence $\left\{I_{i}\right\}$ of nonoverlapping intervals so that $\cup_{i} I_{i}=\cup_{i}\left\langle a_{i}, b_{i}\right\rangle$ and

$$
\left|I_{i}\right|<\frac{1}{2}|I|
$$

Now choose for each $i$ a perfect set $P_{i} \subset h_{1}^{-1}\left(I_{i}\right) \cap F$. Next choose a strictly increasing continuous function $h_{2}$ from $I$ onto itself such that

$$
x \in h_{1}\left(P_{0}\right) \text { implies } h_{2}(x)=x,
$$

$$
h_{2}\left(I_{i}\right)=I_{i}
$$

and $\left|h_{2}\left(h_{1}\left(P_{i}\right)\right)\right|>\frac{1}{2}\left|I_{i}\right|$.

By (2) $x \in P^{0}$ implies $h_{1}(x)=h_{2}\left(h_{1}(x)\right)$. Now put $P^{1}=P^{0} \cup \cup_{i} P_{i}$. Then $P^{1}$ is a perfect set, $P^{0} \subset P^{1} \subset F,\left|h_{2}\left(h_{1}\left(P^{1}\right)\right)\right|>\frac{3}{4}|I|$ and by (2), (3) and (1), $\left|x-h_{2}(x)\right|<\frac{1}{2}|I|$.

Using this method we can define by induction the sequences $\left\{P^{n}\right\}$ of perfect sets and $\left\{h_{n}\right\}$ of strictly increasing continuous functions from $I$ onto itself such that

$$
\begin{array}{ll}
x=h_{2}(x)=h_{3}(x)=\cdots & \text { for } x \in h_{1}\left(P^{0}\right), \\
x=h_{3}(x)=h_{4}(x)=\cdots & \text { for } x \in h_{2}\left(h_{1}\left(P^{1}\right)\right)
\end{array}
$$

and generally,

(4) $x=h_{n+1}(x)=h_{n+2}(x)=\cdots \quad$ for $x \in h_{n}\left(h_{n-1}\left(\cdots h_{1}\left(P^{n-1}\right) \cdots\right)\right)$, 


$$
\begin{aligned}
\left|x-h_{n}(x)\right| & <\left(1 / 2^{n-1}\right)|I|, \\
\left|h_{n}\left(h_{n-1}\left(\cdots h_{1}\left(P^{n-1}\right) \cdots\right)\right)\right| & >\left(2^{n}-1 / 2^{n}\right)|I| .
\end{aligned}
$$

By (5) we have $\left|h_{n}(x)-h_{n+1}\left(h_{n}(x)\right)\right|<\left(1 / 2^{n}\right)|I|$. Hence

$$
\left|h_{n}\left(h_{n-1}\left(\cdots h_{1}(x) \cdots\right)\right)-h_{n+1}\left(h_{n}\left(\cdots h_{1}(x) \cdots\right)\right)\right|<\left(1 / 2^{n}\right)|I| .
$$

By this inequality the sequence of superpositions $\left\{h_{n}\left(h_{n-1}\left(\cdots h_{1}(x) \cdots\right)\right)\right\}$ converges uniformly to a continuous function. Denote this limit function by $\chi$. All $h_{n}$ are strictly increasing so $\chi$ is increasing. By (4),

$$
\chi(x)=h_{n}\left(h_{n-1}\left(\cdots h_{1}(x) \cdots\right)\right) \text { for } x \in P^{n-1} .
$$

Hence $\chi$ is strictly increasing on $P^{n-1}$ and also on the set $\cup_{n=1}^{\infty} P^{n}$ which is dense in $I$. Thus $\chi$ is strictly increasing. By (7)

$$
\chi\left(P^{n-1}\right)=h_{n}\left(h_{n-1}\left(\cdots h_{1}\left(P^{n-1}\right) \cdots\right)\right) .
$$

Hence, since $\chi\left(P^{n-1}\right) \subset \chi(F) \subset I$ by (6) we have $|\chi(E)|>\left(\left(2^{n}-1\right) / 2^{n}\right)|I|$. Hence $|\chi(F)|=|I|$.

This completes the proof of the Lemma.

Proof OF THE TheOREM. Let $\left\{I_{n}\right\}$ be a sequence of all the open intervals with rational endpoints contained in $I$. Since $g\left(I_{1}\right)$ is a subinterval of $g(I)$ and $A$ is dense in $g(I)$, there exists a point $\alpha_{1} \in A \cap g\left(I_{1}\right)$. Let $F_{1}$ be a perfect set such that $F_{1} \subset I_{1} \cap g^{-1}\left(\alpha_{1}\right)$ and $F_{1}$ is nowhere dense in $g^{-1}\left(\alpha_{1}\right)$. Suppose we have chosen for each $i=1,2, \ldots, k$ perfect sets $F_{i}$ and numbers $\alpha_{i}$ such that $F_{i} \subset I_{i}, g\left(F_{i}\right)=\alpha_{i} \in A, F_{i}$ is nowhere dense in $g^{-1}\left(\alpha_{i}\right)$ and the sets $F_{i}$ are disjoint. Let $I^{*}$ be an interval such that $I^{*} \subset I_{k+1}$ and $I^{*} \cap\left(\cup_{i=1}^{k} F_{i}\right)=\varnothing$. Choose a perfect set $F_{k+1} \subset I^{*}$ such that $g\left(F_{k+1}\right)=\alpha_{k+1} \in A, F_{k+1}$ is nowhere dense in $g^{-1}\left(\alpha_{k+1}\right)$. Then $F_{k+1} \cap F_{i}=\varnothing$ for $i \neq k+1$. Clearly the sum $F=\cup_{k=1}^{\infty} F_{k}$ satisfies the conditions of the Lemma. Then there is a homeomorphism $\chi$ from $I$ onto itself such that $|\chi(F)|=|I|$. Denote $H$ $=I \backslash \chi(F), h=\chi^{-1}$ and $\Gamma=\{(x, y): x \in H, y+g(h(x)) \in D\}$. Certainly $\chi(F)$ is a set of the first category of Baire. Thus $H$ is dense in $I$.

If $B$ is a subset of $H \times R$ we shall denote its $x$-projection, i.e. the set $\left\{x: \exists_{y}(x, y) \in B\right\}$ by $\operatorname{dom} B$, and its $y$-projection, i.e. the set $\left\{y: \exists_{x}(x, y) \in B\right\}$ by $\operatorname{rng} B$. For any $t \in H$ we shall denote $B_{t}=\{(x, y): x=t,(x, y) \in B\}$ and for any $s \in R$ we shall denote $B^{s}=\{(x, y): y=s,(x, y) \in B\}$. Since $\Gamma$ is the union of the graphs of all functions $z_{n}-g(h(x))$ where $z_{n} \in D$, it follows that $\operatorname{rng} \Gamma=R$.

We shall say that two points $A\left(x^{\prime}, y^{\prime}\right)$ and $B\left(x^{\prime \prime}, y^{\prime \prime}\right)$ of $\Gamma$ are equivalent, $A \sim B$, if there exists a finite sequence of points $A_{i}\left(x_{i}, y_{i}\right) \in \Gamma, i=1,2, \ldots$, $n$, such that $A=A_{1}, B=A_{n}$, and for each $i$ we have either $x_{i}=x_{i+1}$ or $y_{i}=y_{i+1}$. Denote by $\Gamma / \sim$ the family of all equivalence classes for this equivalence relation. It is clear that for each $t \in H, \operatorname{rng} \Gamma_{t}$ contains a translation of $D$. Then $\operatorname{rng} \Gamma_{t}$ is a dense set. It follows from the definition of 
equivalence that if $G$ is an equivalence class, then $G \cap \Gamma_{t} \neq \varnothing$ implies $G \supset \Gamma_{t}$. In the same way if $G \cap \Gamma^{s} \neq \varnothing$ then $G \supset \Gamma^{s}$.

Now we shall prove that all dom $\Gamma^{s}$ are dense in $H$. Since $g$ is not constant on any interval then for any open interval $I^{*} \subset I$ there exist points $x^{\prime}$ and $x^{\prime \prime}$ in $I^{*}$ for which $g\left(h\left(x^{\prime}\right)\right) \neq g\left(h\left(x^{\prime \prime}\right)\right)$. Since $D$ is dense there exists an $r \in D$ such that $r-s$ lies between $g\left(h\left(x^{\prime}\right)\right)$ and $g\left(h\left(x^{\prime \prime}\right)\right)$. Since $g \circ h$ is Darboux there exists an $x \in I^{*}$ for which $g(h(x))=r-s$. If $x \in H$ then $(x, s) \in \Gamma$ and therefore $x \in \operatorname{dom} \Gamma^{s} \cap I^{*}$.

If $x \notin H$ then $x \in \chi(F)$. Thus $r-s=g(h(x))=g\left(\chi^{-1}(x)\right)=\alpha_{i} \in A$. The level set $g^{-1}\left(\alpha_{i}\right)$ is a perfect set and $h(F) \cap g^{-1}\left(\alpha_{i}\right)=h\left(F_{i}\right) \cap g^{-1}\left(\alpha_{i}\right)$. The set $h\left(F_{i}\right)$ is nowhere dense in $g^{-1}\left(\alpha_{i}\right)$. It follows that there exists a point $x^{*} \in I^{*} \cap\left[g^{-1}\left(\alpha_{i}\right) \backslash h(F)\right]$. Thus $x^{*} \in H \cap I^{*}$ and $\alpha_{i}=g\left(h\left(x^{*}\right)\right)=r-s$. Hence $\left(x^{*}, s\right) \in \Gamma$ and $x^{*} \in \operatorname{dom} \Gamma^{s} \cap I^{*}$. This shows that dom $\Gamma^{s}$ is dense in $H$.

Suppose $A\left(x^{\prime}, y^{\prime}\right) \sim B\left(x^{\prime \prime}, y^{\prime \prime}\right)$. Then

$$
y^{\prime \prime}-y^{\prime}=\sum_{i=1}^{n-1}\left(y_{i+1}-y_{i}\right)=\sum_{i^{\prime}}\left(y_{i^{\prime}+1}-y_{i^{\prime}}\right)
$$

where $1 \leqslant i^{\prime} \leqslant n$ and $y_{i^{\prime}+1}-y_{i^{\prime}} \neq 0$. This inequality implies $x_{i^{\prime}}=x_{i^{\prime}+1}$. Then

$$
y_{i^{\prime}+1}-y_{i^{\prime}}=y_{i^{\prime}+1}-g\left(h\left(x_{i^{\prime}+1}\right)\right)-\left(y_{i^{\prime}}-g\left(h\left(x_{i^{\prime}}\right)\right)\right) \text {. }
$$

So $y_{i^{\prime}+1}-y_{i^{\prime}}$ is the difference of two members of $D$. The set of all such differences is countable. The set of finite sums of these differences is countable too. Each difference $y^{\prime \prime}-y^{\prime}$ is such a sum of this form. Therefore the set of all differences of ordinates of points belonging to an equivalence class $G$ is countable. Thus $\operatorname{rng} G$ is countable. Let us enumerate members of $\operatorname{rng} G$ as $\left\{y_{m}\right\}$ and all rational subintervals of $I$ as $\left\{J_{n}\right\}$. Now enumerate all pairs $\left(y_{m}, J_{n}\right)$ as $\left\{\left(y_{m_{i}}, J_{n_{i}}\right)\right\}$. Since $\operatorname{dom} \Gamma^{y_{m_{1}}}$ is dense in $H$, there exists a point $t_{i} \in J_{n_{1}}$ $\cap \operatorname{dom} \Gamma^{y_{m_{1}}}$. Let $t_{1}, t_{2}, \ldots, t_{k}$ be chosen such that $t_{i} \in J_{n_{i}} \cap \operatorname{dom} \Gamma^{y_{m_{i}}}$ and $t_{i} \neq t_{j}$ for $1 \leqslant i<j \leqslant k$. Take $t_{k+1} \in J_{n_{k+1}} \cap \operatorname{dom} \Gamma^{y_{m_{k+1}}}$. Each set $\operatorname{dom} \Gamma^{y_{m}}$ contains a dense set of members of the sequence $\left\{t_{n}\right\}$. Set $d(x)=y_{m_{i}}$ for $x=t_{i}$. Then $\left\{d(x): x=t_{n} \in J_{m}\right\}=\operatorname{dom} G$. Since $\cup_{G \in \Gamma / \sim} \operatorname{rng} G=R$ the function $d$ defined on $\cup_{G \in \Gamma / \sim}\left\{t_{n}\right\}$ maps each intersection $H \cap J_{m}$ onto $R$. If $G^{\prime} \neq G^{\prime \prime}$ then $\operatorname{dom} G^{\prime} \cap \operatorname{dom} G^{\prime \prime}=\varnothing$. Therefore there is no contradiction in our extension of $d$ from the subset of $\operatorname{dom} G$ to the sum $\cup \operatorname{dom} G$.

At present $d$ is defined on a subset of $H$. This subset is dense in $I$, therefore each next extension of $d$ on $I$ must be Darboux. Let us fix a point $c \in D$. Set $d(x)=c-g(h(x))$ for each point $x$ at which we have not defined $d(x)$. Since $|H|=0, g(h(x))+d(x)=c$ almost everywhere.

We still have to prove that $d-(g \circ h)$ maps $I$ onto $D$. Let $G^{0}$ be the equivalence class containing $\Gamma^{0}$. Then $D \in \operatorname{rng} G^{0}$. For each $x=t_{n}$ the point $(x, d(x))$ belongs to $\Gamma$. Thus $d(x)+g(h(x)) \in D$. For each $x \neq t_{n}$ we have 
$d(x)=c-g(h(x))$ where $c \in D$ so that $d(x)+g(h(x)) \in D$. This completes the proof of Theorem.

\section{REFERENCES}

1. A. M. Bruckner and J. Ceder, On the sum of Darboux functions, Proc. Amer. Math. Soc. 51 (1975), 97-102.

2. K. M. Garg, Monotonicity, continuity and level sets of Darboux functions, Colloq. Math. 28 (1973), 91-103. MR 48 \#2317.

3. J. S. Lipiński, On level sets of Darboux functions, Fund. Math. 86 (1974), 193-199. MR 50 \# 10170.

4. A. N. Singh, On some new types of non-differentiable functions, Ann. of Math. (2) 28 (1927), 472-476.

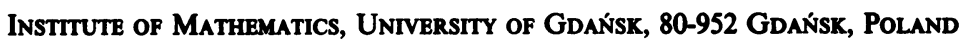

